\title{
Changes and Trends of Product Identification and Design in the Context of Internet
}

\author{
Pengfei $\mathrm{Li}^{\mathrm{a}}$ and Xufeng Cheng ${ }^{\mathrm{b} \text { * }}$
}

Beijing Forestry University, Beijing 100083, China
${ }^{a}$ anita0826@126.com, ${ }^{b^{*}} 1141513971 @ q q . c o m$

Keywords: Product identify design; Industrial design; Internet; Ecological concept; Design Flow

\begin{abstract}
In the Internet age, smart links between products form the Internet of Things (ie, the product ecosystem), and the influence of the ecological principles of the natural world on the product ecosystem is gradually revealed. Product Identity (PI) designs also carrying out the inheritance and development similar to biological genetics. Through the research and analysis of the characteristics of the era of the Internet and the guidance of the ecological concept, the article explores and summarizes the changes in product design (PI) to design process and overall development trends, and presents a plan for the development of a new PI design concept. The study of developmental changes provided a reference.
\end{abstract}

\section{Introduction}

With the advancement of science and technology and the acceleration of social development, the current full coverage of the Internet, products and products began to be interconnected by the network as a medium. The spread and visualization of the Internet has refocused the attention from companies, designers and users on the importance of product identification (PI) design. This article mainly discusses the future development of product identification and design concept, flexible application of design flow and design practice under the background of the Internet era with sustainable development as the theme.

\section{Research Background of Product Identification in the Internet Age}

The Social Background of Product Design in the Internet Age. Under the background of the Internet era, industrial design has become an important driving force for China's economic transformation and development. Product designs focuses on the human-centered approach from meeting functional requirements. In the product design mode of the Internet era, industrial products pass the Internet, big data and clouds. Calculations, etc. be linked to other data terminals. Therefore, the design of industrial products of the Internet environment must be user-centric. In recent years, big data has provided users with more choice opportunities, and Internet companies have rapidly emerged. Compared with the traditional process of R\&D of products by traditional enterprises, the new structure of the lightweight, flexible $R \& D$ team of new small and medium-sized enterprises is focused on users to conduct research and design. With the rapid product updates, strong independent research and development capabilities, and innovative design and many other advantages, products serve as a medium for companies and users to communicate. They not only shoulder the concept of corporate culture, the symbol of corporate capabilities, but also met the individual needs of users. [1]

The Social Background of PI (Product Identification) Design in the Internet Age. Product Identity (PI for short) originated from the system design promoted by the Ulm School of Modelling in Germany in the 1950s. It is an important carrier for corporate culture and is one of the main design methods of product differentiation. The main role are reflected on the close relationship among products, users, and businesses. In the Internet era, products form their own " eco-environment " in the Internet of Things environment. The influence of nature's ecological principles of the product ecosystem is also gradually revealed. PI designs carry out inheritance and development similar to biological inheritance under the guidance of the design and evolutionary theory of natural sciences. For example, the image positioning of the enterprise product groups of famous brand products such as Braun Electric Appliance and BMW Motors in the last century is a prominent case of the application of product identification design, and is 
called Product Family. In general, product family is the design of product image and related products based on product image by a series of related marketing activities and based on the characteristics of the image of the company's products [2]. From the perspective of the natural sciences, organisms maintain the unity of the population to some extent through the inheritance and expression of DNA. At the same time, due to the presence of gene mutations in the genetic process, there are always differences between individuals. Genes control the characteristics of individual organisms by directing the synthesis of proteins to express their own genetic information [3]. When companies design products, they want to make the product groups show certain similar or uniform characteristics. In due course, companies will use the role of " genes " to guide the formation of a family product image.

The production of a family of products is to a certain extent the saving of costs and the unified control of product visualization. It is also a commercial means of improving product categories under the limited conditions-reusing the same technologies and processes[4]. In the era of user-centric Internet, along with the design criteria and high standards requirements, and also to urge the enterprise to systematically plan the product design, the product identification system began to be incorporated into the design process and enterprise management. The areas involved in product design is also becoming wider and wider. Design activities are increasingly systematic. Product identification planning and design developed under the guidance of the core content of product identification has become a new trend . [5]

\section{Current Status of Product Identification (PI) Design}

Product Identification (PI) Design Status. Product identification design are mainly to guide users' attention to corporate brands of products, so that users have an emotional connection with brands and bring greater benefits to the company. With the expansion of the coverage of the Internet and the improvement in the timely sharing of scientific and technological information, the differentiation of similar products is not obvious. How to create a unique identification factor for an enterprise is an important issue for designers. Including product styles, differentiated design between competing products will become the main entry point[6] .

In the fierce market competition environment, if companies want to attract users ' attention to products and establish a relationship of identifying information on users, leaving a unique corporate image of users, they are required to have a product design strategy that can express themselves independently-which let the product become the company's "spokesperson ". In the early stage of product identification design, the company's concept can be regarded as the actual product modeling style, but with the change and promotion of product design requirements, all aspects of the factors affect the transition to conceptual to strategic. In the process of the company's own development, the design gradually penetrated into the management of the enterprise, at the same time, the change of the company also made the product design became more systematic [7]. Therefore, products that are limited to analysis and extend in the similarity and diversity of current products do not solve the problem of serialized product group identification pursued by the company, nor do it satisfy the "sustainable development " plan for enterprise product identification. The long-term development is also unfavorable.

Product Identification (PI) Design Process Development. In the process of brand building, it is crucial to companies to convey the company's philosophy culture to users by strengthening product identification and design. Therefore, the application and research of product identification of design are a macroscopic and important part of sustainable development [8]. Foreign companies such as Apple Inc. and BMW Corporation had early research on product identification (PI) design and achieved remarkable results, and spread influence through multiple aspects and levels which let many domestic companies to realize the importance of product identification (PI ) design. Integrate product identification (PI) into corporate R\&D strategy. Since the advent of the PI design concept, the company has made step-by-step product identification designs to make the product more characteristic of the company itself and form a family. Companies make differentiated designs combine with the characteristics of competing products. After 4 stages of conceptual analysis, design gene extraction, 
optimization, and finalization, they can find out suitable product family design genes through multi-dimensional analysis . [9]

\section{Trends in Product Identification (PI) Design}

The research of product identification (PI) designs mainly focuses on product family DNA design, product image research and design, enterprise specific product PI research, and PI design prototype design system, and forms a certain process applied to the enterprise PI design (such as Figure 1 ).

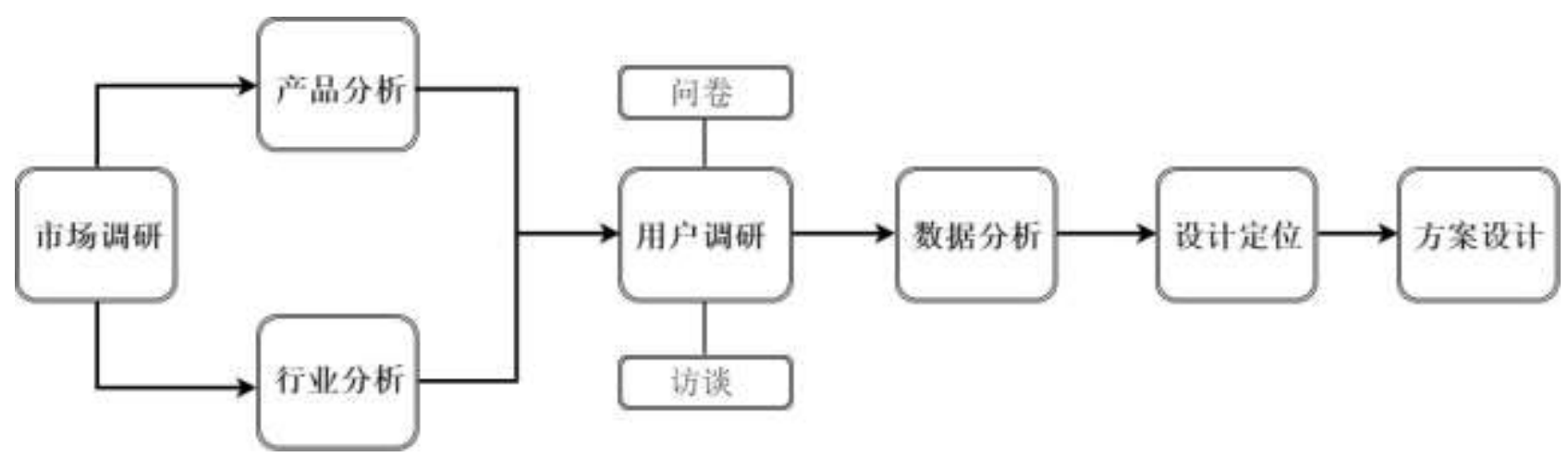

Figure 1. The general process of PI design

Currently, with the influence of the Internet environment, sustainable development, ecological concepts, user experience, and product iterations strengthening, PI design needs to add research content in these areas and iteratively generate a new PI design process (Figure 2). Mainly in the following eight areas:

(1) The enterprise's positioning analysis of its own brand product attributes. Before carrying out PI design for an enterprise, it is necessary to analyze the core design concepts of the enterprise first, in order to guide the enterprise to carry out the PI design process. It should be note that the company's core design philosophy runs through the entire PI design process. In the future, the PI design concept can fully understand the company's design trends and brand values "through various methods such as industry research, product analysis, and interviews. The original visual symbols (VI) of the enterprise, such as the design, color, material, and shape of the company's LOGO, can also be applied to the element extraction process of PI. All the planation and visual elements of the enterprise can be integrated to strengthen the recognition degree and relevance between corporate image and the company's products. At the same time, the different elements from the selected company's existing products elements should be resorted so that they can be flexibly used and modified in future PI design concepts and product iterations. 


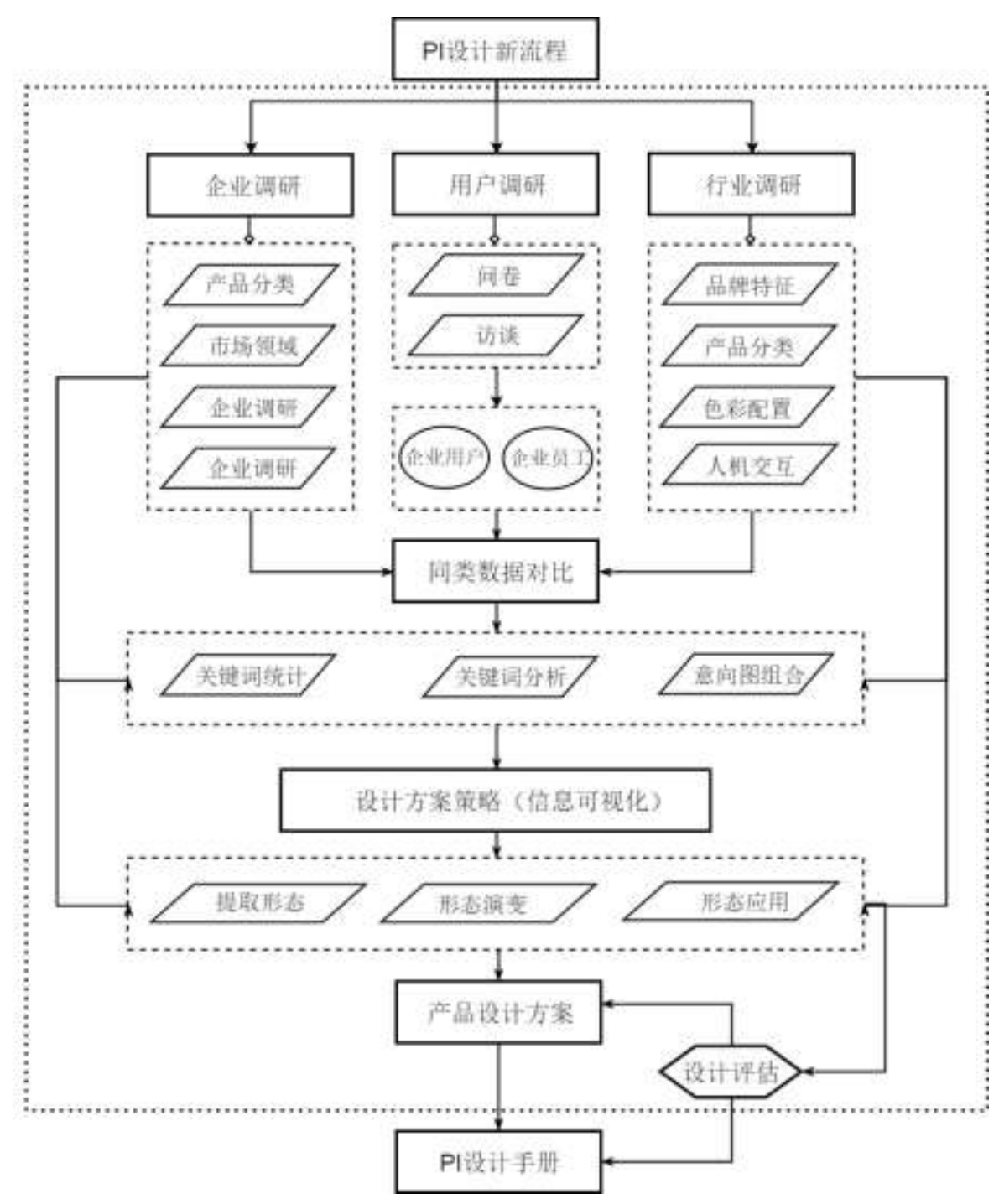

Figure 2. PI design new process architecture

(2) Research and analysis on the industry and competing products. The preliminary research of the enterprise is conducted through the three parts of investigation and analysis, product analysis of the enterprise, and user research. The company's research and analysis contents mainly revolve around the characteristics of the enterprise, the current product classification of the enterprise, product iteration, and the scope of the enterprise market. The enterprise product analysis mainly analyzes the information about the enterprise products from many aspects such as the shape of the product, color configuration, and human-machine interaction. For industry competition products, they are selected through online data and field research methods. This section uses the same method to analyze the selected competitive products. After that, the above sections are combed to analyze. through the way of information visualization or intention maps and imagery vocabulary from four categories of brand positioning, design elements, design colors, and human-computer interaction, in order to obtain the comprehensive rule of industry.

(3) The method of enterprise-to-user analysis. The analysis of business-to-user (including corporate employees and corporate customers) mainly focuses on user psychology and behavior identification through questionnaires, interviews, and other forms to analysis. The behavioral identification of product users is mainly to study the dynamic behavior between the two, using scenario analysis, to establish the identification features through these interactive behavioral characteristics of users, and to apply the features to future products. The preset use of a good product should meet the user's behavioral characteristics criteria, study the user's perception of the product, operation and cognitive behavior, and use this as a basis for the behavior identification design. [10] 
(4) Summarize keywords, information visualization/imagery analysis. The keyword decomposing is not only to sort out the previous research data in time, but also to obtain a number of image maps and design intention analysis. Through discussion and analysis, four groups of first-level keywords are sorted out, and the set design directional keywords are secondarily decomposed to finally obtain a number of three-level designs directional keywords. The product images elements here include four aspects related to design, material technology, engineering design, and user experience. Through the Internet or shooting, etc, the image map corresponding to these three levels of key words is obtained, and the abstract vocabulary is converted into more intuitive information data and pictures. Through the method of establishing the coordinate quadrant, four groups of first-level keywords are developed, four groups of image maps are placed in the corresponding regions, and certain regions are selected to obtain the design keyword tendency.

(5) Extract the basic form. In the previous design strategy summary, the analysis of a number of corresponding intention map, according to the actual situation of the enterprise product design, some of the basic form elements that are specific design elements can be extracted from and be used in product design, in order to facilitate the later product changes.

(6) Evolution and application of basic morphology. Through the above information, compare, summary and re-evaluate the existing products of the company.. For the basic line types of the products and the traditional lines that continue to be used, they are selective reserved or discarded. The graphical features of the redesigned and evolved products are used as the main identification features. The unified line features are extracted from the significant design features existing on similar functions according to functional analysis, which will make the enterprise PI plan reflect a unified brand image of both historical dimension and product dimension, which is beneficial to brand growth. [11]

(7) Display of PI manual results. The PI Handbook summarizes and refines the identification elements in the completion or ongoing of product identification design. It also summarizes and displays the results of the entire PI design process. The construction of PI manual is to provide the theoretical basis and support for product identification design practice. It not only guide the product identification design smoothly and accurately, but also technically guarantee the sustainability of product identification elements, and guide enterprises to design in product modeling, color, material, human-computer interaction and other aspects.

(8) PI designs assessment. When carrying out product identification design, it is necessary to evaluate repeatly each key link in the process to ensure the consistency of the product image of the company and the accuracy of the data. At the same time, when carrying out design evaluation, it is necessary to provide relevant standards and questionnaires for the enterprise's design department as a reference, to conduct a comprehensive quantitative assessment of the final design of the product.

\section{Product Identification (PI) Design Application Research - Taking Ningxia Power Products as an Example}

Basic Process. First, get the company's existing product features and brand characteristics from the company's own product research and analysis. Determine the company's core concepts and needs, and then from the analysis of the industry's overall situation and user research to obtain the needs of consumers and the company's own product expectations. Based on the above analysis result of the main body to determine the company's PI design philosophy. Show mainly in the form of design directional keywords, give these keyword intention maps, establish quadrant maps for image map combination, select the design strategy, and carry out specific program design. And in the design process, design concept has always run through the entire process, through the results of previous research and analysis and the user's needs analysis, enter the design process, and finally come to the design program.

Process Introduction and Effect Display. (1) Based on the previous research and discussion of the company's products and brands, find and concise the four design direction keywords: solid, honest, fine, and extensive. Decompose the keywords into product images elements, including four aspects related to design, material technology, engineering design, and user experience. 
(2) In order to improve the adaptability of PI elements to achieve the goal of combining the use of different functional components and other elements, after repeated analysis and study and comparison, it is determined that the part of the extracted company's LOGO (logo) element is the core PI element, such as See Figure 3.

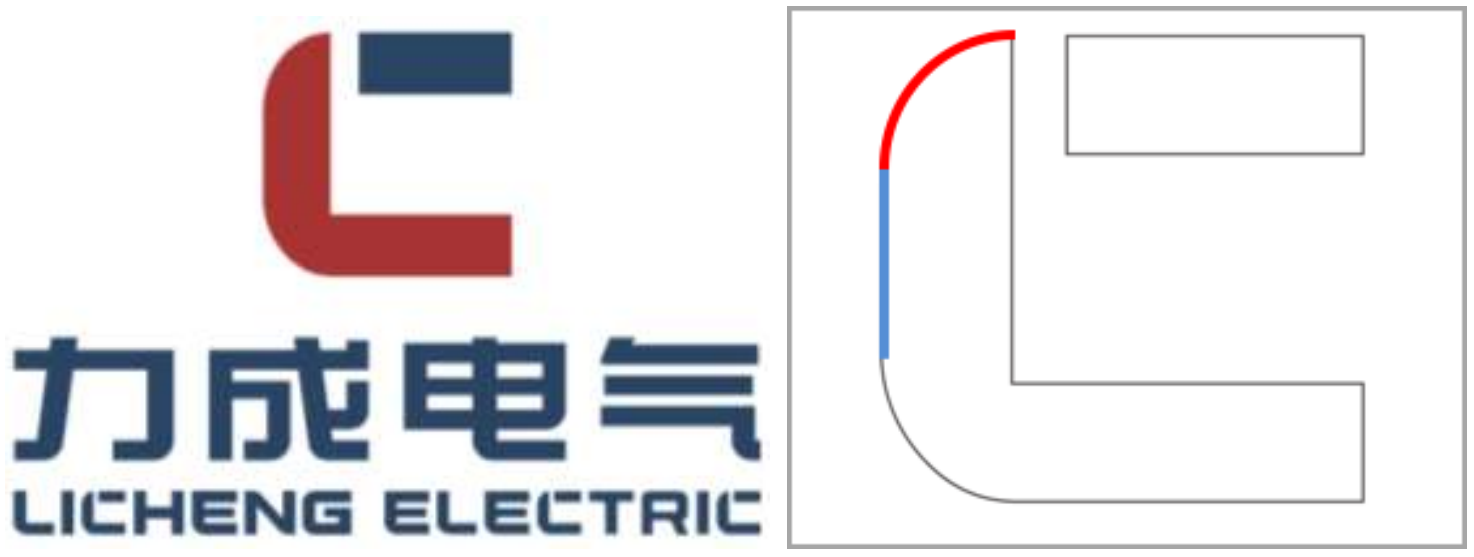

Figure 3. Company logo and local elements

(3) Considering the overall modeling style of Licheng products and the regularity of product designs, the main PI elements are " arcing ", " straight line, " square ", " C letters / lettered ", " circle " and " plotline ". As shown in Figure 4. Using these extracted styling elements need strictly follow the deformation rule of the elements, and zoom the overall dimensions in proportion, stretch the part of straight line horizontally or vertically according to the styling size ,prohibits arbitrary cutting or twisting. As shown in Figure 5.
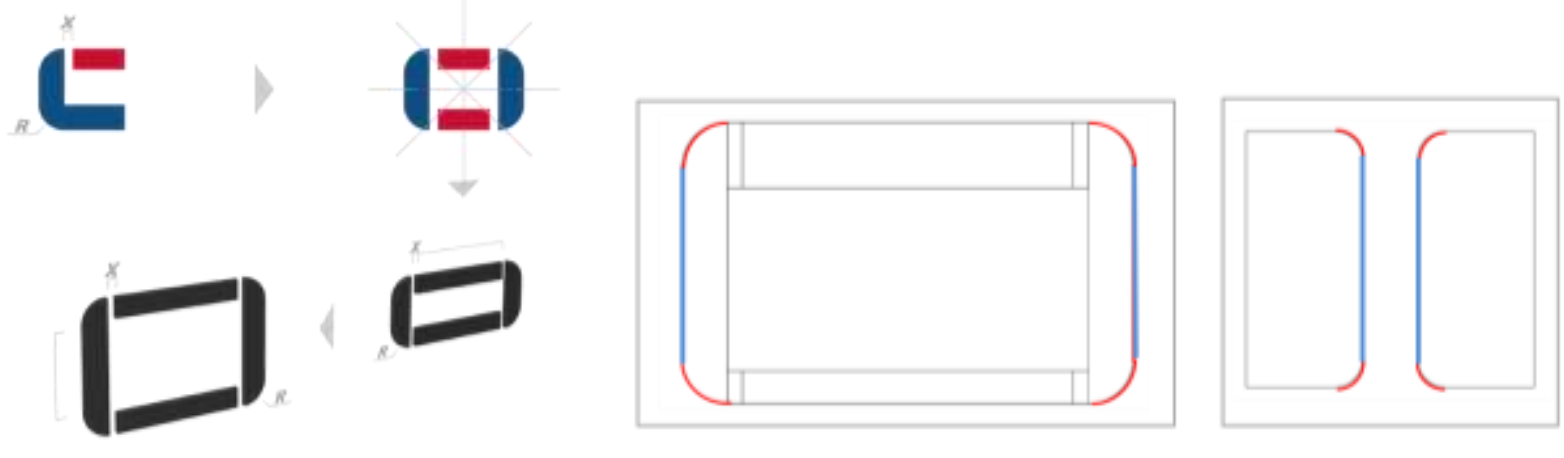

Figure 4. LOGO element deformation and application 

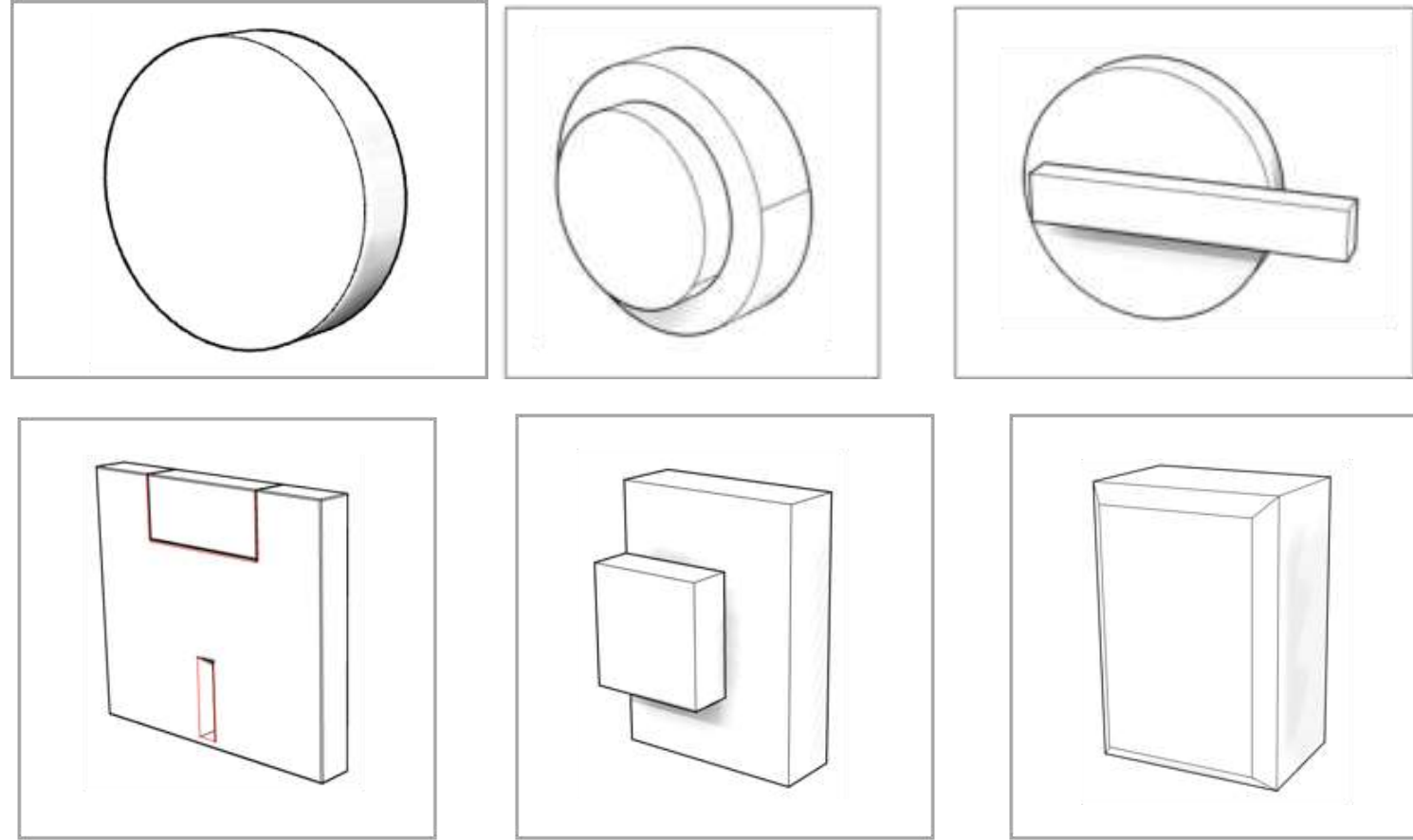

Figure 5. Basic elements

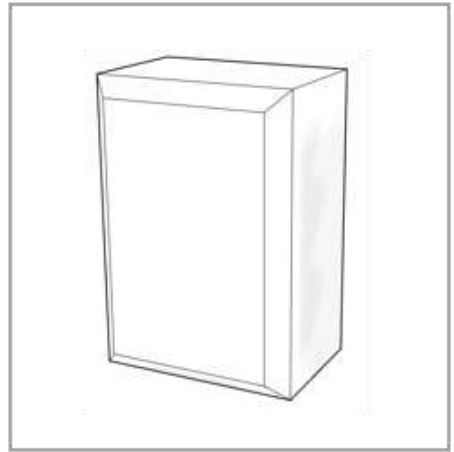

(4) According to the above product design plan strategy for enterprise product PI design program, as shown in Figure 6.

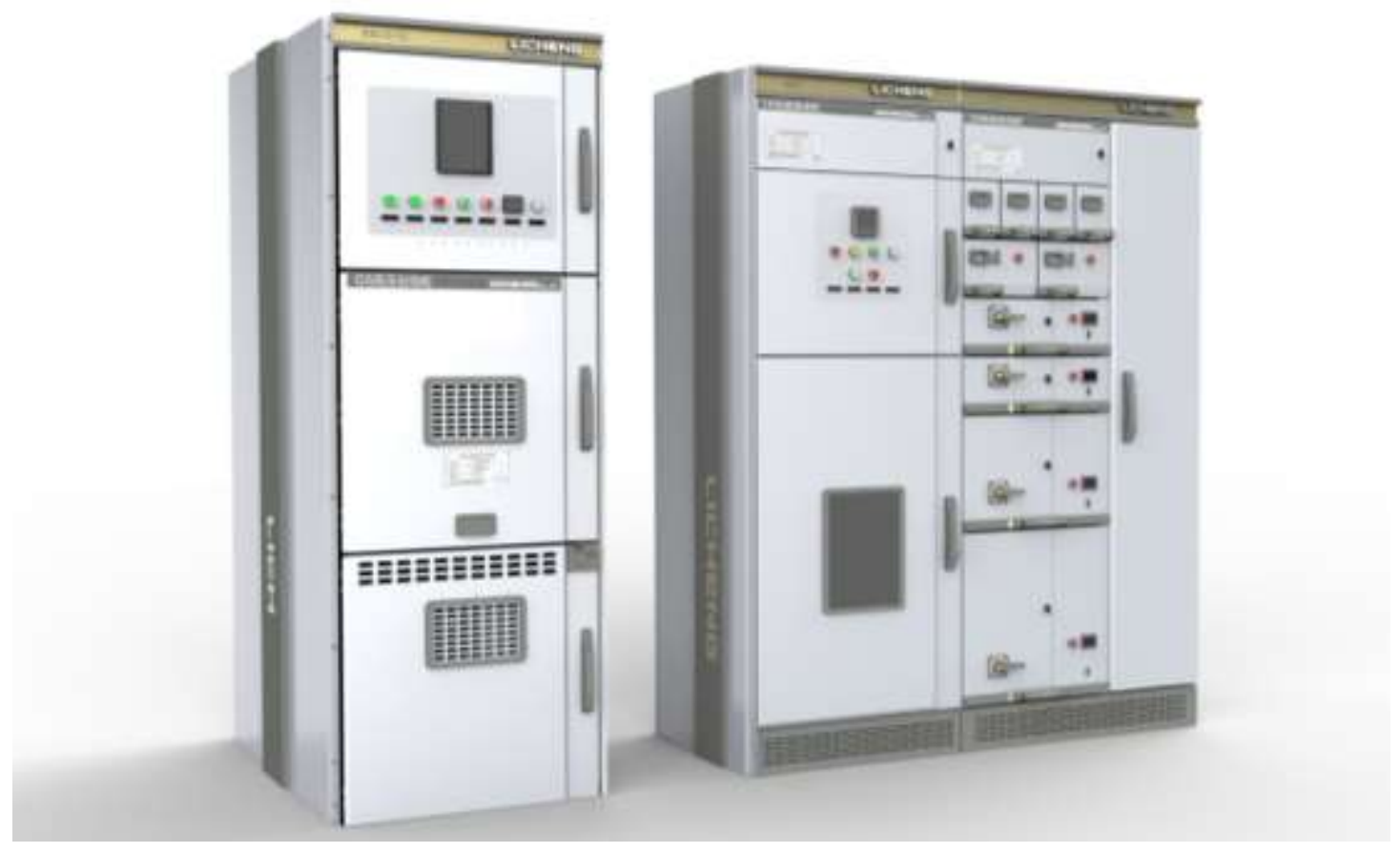

Figure 6. Design scheme of medium and low voltage distribution cabinet

\section{Conclusion}

Based on the Internet age, through the analysis of the current situation and changes of PI design, this study summarized the development trend of PI designs. The design flow and design method of PI design are analyzed, summarized to provide guidance for product identification design. At the same time, research results have verified through design practice, PI design tasks with distinctive brand personality 
and high recognition are completed, and finally the purpose of product identification is improved, which provide specific method reference and exploration for product identification design.

\section{Acknowledgements}

The authors are grateful for the support of the Fundamental Research Funds for the Central Universities (No. 2017ZY72).

\section{References}

[1] L.Liu,X.Q.Fu and M.Kang.Research on Product Modeling DNA Design of Small and Medium-sized Enterprises for Brand Identification[J].Machine Design,2014,31(09):105-108. ( in Chinese) .

[2] Z.S.Zhang, X.F.Cheng. Study on lawn mower design based on brand family[D]. Beijing Forestry University, 2018. ( in Chinese) .

[3] Mozota B B. Design management using to build brand value and corporate innovation[M]. New York: All Worth Press, 2013.

[4] W. Johannsen. Elemente der exakten Erblichkeitslehre. Gustav Fischer, Jena, 1909.

[5] Z.B.Tian. Research on appearance design of smart home appliances based on brand product identification [D]. East China University of Science and Technology, 2014. ( in Chinese) .

[6] X.X.Yang , X.M.Wang . Identification of product family design from the perspective of ethnic culture [J]. Art and Design (Theory), 2017, 2(08): 86-88.( in Chinese) .

[7] S.W.Zhang, X.F.Cheng. Study on the Influence of Ecological Ideas on Product Identity Design in Internet Age[C]// International Conference on Social Network, Communication and Education. 《Advances in Computer Science Research Vol.82》2017.501-508.

[8] X.W.Li . Research on Application of Product Identification Design [D]. Nanjing Art Institute, 2011. ( in Chinese) .

[9] S.J.Luo, S.S.Zhu, F.T.Ying, et al. Gene design based on vision-behavior-emotional product family [J]. Computer Integrated Manufacturing Technology, 2009, 15(12). ( in Chinese) .

[10] W.Huang, X.Huang, Q.X.Qiao. Behavior Identification of Product Identification Design [J]. Packaging Engineering, 2016, 37(22): 114-118. ( in Chinese) .

[11] Z.T.Tan, J.H.Zhao. Brand Product Identification Design Based on Line Features and Graphic Features [J]. Packaging Engineering, 2014, 35(24): 17-21. ( in Chinese) . 